\title{
The Role of Cytokines in Predicting the Efficacy of Acute Stage Treatment in Patients with Schizophrenia
}

This article was published in the following Dove Press journal: Neuropsychiatric Disease and Treatment

\author{
Xiaoyan $\mathrm{He} \mathbb{D}^{\prime}$ \\ Qingyan $\mathrm{Ma}^{\mathrm{I}}$ \\ Yajuan Fan' \\ Binbin Zhao' \\ Wei Wang' \\ Feng Zhu ${ }^{2}$ \\ Xiancang $\mathrm{Ma} \mathbb{D}^{\prime}$ \\ Lina Zhou' \\ 'Department of Psychiatry, The First \\ Affiliated Hospital of Medical College of \\ Xi'an Jiaotong University, Xi'an, Shaanxi, \\ People's Republic of China; ${ }^{2}$ Center for \\ Translational Medicine, The First \\ Affiliated Hospital of Xi'an Jiaotong \\ University, Xi'an, Shaanxi, People's \\ Republic of China
}

Correspondence: Lina Zhou

Department of Psychiatry, The First

Affiliated Hospital of Medical College of

Xi'an Jiaotong University, Xi'an, Shaanxi,

People's Republic of China

Email zhoulinapsy@xjtu.edu.cn
Purpose: Inflammatory response in schizophrenia $(\mathrm{SCz})$ is related to its underlying pathological mechanism and might be significant in deciding a patient's prognosis. The current study aims to investigate the differences in the serum inflammation level between schizophrenic patients and healthy controls and identify inflammatory markers that can predict clinical therapeutic effects in early-stage $\mathrm{SCz}$ patients at the 6-month follow-up.

Patients and Methods: In total, 71 subjects were recruited in this study, including 35 patients with Scz and 36 healthy controls. The $35 \mathrm{Scz}$ patients, who were in the first-episode or acute relapse state at admission, had completed the 6-month follow-up. The Positive and Negative Syndrome Scale (PANSS) and the Clinical Global Impression (CGI) assessment results, demographic details, and blood samples were collected at the baseline and at followup. Data were analyzed using the Spearman correlation and multiple linear regression.

Results: Serum interleukin (IL-1 $\beta$, IL-4, IL-6, and IL-8) levels were significantly elevated in $\mathrm{SCz}$ patients at baseline compared with healthy controls, with a reduced IL-8 level at the follow-up. Furthermore, a higher IL-6 level and lower IL-8 level was found to predict better improvement in negative symptoms. The higher IL-6 level also predicted lesser improvement in depressive symptoms. Finally, a higher interferon (IFN)- $\gamma$ level predicted a lower therapeutic effect for excitatory symptoms.

Conclusion: The serum levels of inflammatory markers were higher in patients with $\mathrm{SCz}$ than in healthy controls. These markers can be considered accurate predictors of therapeutic effects in patients with SCz.

Keywords: schizophrenia, cytokines, therapeutic effect, IL-6, IL-8

\section{Introduction}

Schizophrenia $(\mathrm{SCz})$ is a heterogeneous multi-factorial illness with a lifetime prevalence of approximately $1 \%$ in the worldwide adult population. ${ }^{1,2}$ This severe mental disorder generally begins in the early adolescent years and is characterized by "positive", "negative", and "cognitive" symptom categories. ${ }^{3}$ Schizophrenic patients have a 2.6-fold increased risk of death than the normal population. ${ }^{4}$ It is interesting to note that in the total recorded duration of life with disability measured as 13.4 million years, a significant global burden was due to $\mathrm{SCz}$, which was a huge cost to society. 5,6 Therefore, some scholars have suggested that it is important to study the influencing factors that may predict the level of disability in $\mathrm{SCz}$ and create effective interventions that are aimed at disability reduction. ${ }^{7,8}$ Recent studies have dealt with improving the prognosis prediction of $\mathrm{SCz}$ by the use of circulating biomarkers, for example, serum 
proteins ${ }^{9}$ and C-reactive protein. ${ }^{10}$ However, no study has, to date, explored the effect of peripheral blood cytokine levels on the progress of $\mathrm{SCz}$.

The incidence of $\mathrm{SCz}$ is associated with infections ${ }^{11}$ and autoimmune conditions ${ }^{12}$ and probably involves the inflammatory immune response pathway. ${ }^{13}$ Recent evidence from genetic studies suggests that $\mathrm{SCz}$-associated loci include multiple genes that encode for the immune system. ${ }^{14}$ Cytokines may contribute to the psychopathology of SCz through an inflammatory immune response that can affect neurodevelopment, synaptic plasticity, and neurotransmission. ${ }^{15}$ Numerous clinical studies have found that the cytokines, such as interleukin (IL)-1 $\beta$, IL-6, IL-8, IL-12 p70, interferon (IFN)- $\gamma$, and tumor necrosis factor (TNF)- $\alpha$, showed varying levels between $\mathrm{SCz}$ patients and healthy subjects. ${ }^{16-18}$ As inflammation becomes widely confirmed as an essential process in the development of $\mathrm{SCz}$, an increasing volume of literature suggests that antiinflammatory treatment, such as the administration of cyclooxygenase (COX) inhibitors ${ }^{19}$ and acetylsalicylic acid (ASA), ${ }^{20}$ is beneficial in the treatment of SCz. Moreover, researchers have documented positive effects of such treatments using the total scores of the Positive and Negative Syndrome Scale (PANSS).

While many studies have shown that the immune system can be considered a novel therapeutic target for $\mathrm{SCz}, 7,8,19,20$ there is a paucity of research that explores whether the baseline serum cytokine levels are able to predict $\mathrm{SCz}$ outcomes or not. Therefore, we conducted a 6-month long clinical cohort study to identify possible bio-predictors that could accurately predict a treatment response for $\mathrm{SCz}$ patients. In terms of the cytokine measurements, we measured inflammation-associated serum cytokine levels using Luminex technology instead of the classic enzyme-linked immunosorbent assay (ELISA) method, because the Luminex assay platform has higher sensitivity, accuracy, and precision than ELISA. ${ }^{21}$ We aim to confirm the difference of serum cytokine levels between the SCz patients and healthy controls and explore the link between the baseline serum cytokine levels and the treatment efficacy of the patients after they received a course of therapy.

\section{Methods}

\section{Participants}

The SCz patients were recruited at the psychiatry department of the First Affiliated Hospital of Xi'an Jiaotong University. All 35 patients met the following inclusion criteria: (1) had $\mathrm{SCz}$ according to the criteria of the Diagnosis and Statistical
Manual of Mental Disorders, Fourth Edition (DSM-IV) as confirmed by two independent experienced psychiatrists; (2) were aged 16-50 years; (3) had Positive and Negative Syndrome Scale (PANSS) scores greater than 60; (4) had $\mathrm{SCz}$ in the first-episode or acute relapse state; (5) had no current allergies, autoimmune diseases, or infections; (6) were not taking immunosuppressants or anti-inflammatory drugs; (7) had been taking antipsychotic drugs for less than two weeks and not reaching the effective dose (first-episode patients); and (8) chronic patients who had stopped taking antipsychotics more than 3 months before and the disease had not lasted longer than 3 years for those in an acute relapse state. The inclusion criteria for healthy controls were: (1) no personal or family history of mental disorder; (2) no history of any neurological diseases or traumatic brain injury; (3) no current allergies, autoimmune diseases, or infections; and (4) free of immunosuppressants or anti-inflammatory drugs.

We recruited 36 healthy subjects from the general population using advertisements. These controls were screened to rule out infectious or inflammatory diseases and other mental disorders, except SCz. All the patients were treated at the same center and received a complete medical history collection, physical examination, and clinical evaluation. These treatments were conducted on the first day of hospital visit and at the 6-month follow-up. All the patients had been receiving atypical antipsychotic treatment, including risperidone $(n=15)$, clozapine $(n=1)$, olanzapine $(n=3)$, amisulpride $(\mathrm{n}=1)$, haloperidol $(\mathrm{n}=1)$, risperidone and amisulpride $(\mathrm{n}=5)$, risperidone and haloperidol $(\mathrm{n}=5)$, risperidone and aripiprazole $(n=3)$, and olanzapine and aripiprazole $(n=1)$. This study was conducted in consistent with the declaration of Helsinki following the ethical standards for human research. Each participant gave a written informed consent after having received a detailed explanation of the study. Participants who were under the age of 18 were provided with written informed consent from their parent or legal guardian. This study was approved by the Ethics Committee of The First Affiliated Hospital of Xi' an Jiaotong University (No. XJTU1AF2015LSL-079).

\section{Assessment of Patients}

The Positive and Negative Syndrome Scale (PANSS) and the Clinical Global Impression (CGI) were administered by a clinical psychiatrist to evaluate the symptom severity of patients with $\mathrm{SCz}$. We analyzed the data using 5-factor model of PANSS ${ }^{22}$ and measured the therapeutic effect using the reductive ratio of PANSS total scores, PANSSpositive scores, PANSS-negative scores, PANSS-cognitive 
scores, PANSS-excited scores, and PANSS-depressed scores. The reductive rate $(\mathrm{R})$ was calculated by assessing the SZ baseline (B) and the follow-up (F) PANSS scale scores. The equation was $\mathrm{R}=(\mathrm{B}-\mathrm{F}) / \mathrm{B}$.

\section{Cytokine Measurements}

We collected venous blood samples from all subjects on the second day of their hospital visit, in the morning after a confirmed overnight fast. A second blood sample was collected from 35 patients at the 6-month follow-up after an overnight fast. On the same day, the PANSS and CGI assessment were performed. The blood samples were collected in $4 \mathrm{~mL}$ plain tubes (without anticoagulation) and centrifuged at $3000 \mathrm{rpm}$ for $10 \mathrm{mins}$. The serum was separated and stored at $-80^{\circ} \mathrm{C}$ until it was ready for cytokine assessment. All the assays were performed by the same technician using the Luminex bead-based immunoassay (R\&D Systems, LXSAHM-09, Minneapolis, US) to assess the levels of inflammatory cytokines, which included interleukin (IL)-1 $\beta$, IL-4, IL-6, IL-8, IL-12p70, tumor necrosis factor (TNF)- $\alpha$, interferon (IFN)- $\gamma$, and C-C motif chemokine ligand (CCL)-2. Their sensitivities were $0.8 \mathrm{pg} / \mathrm{mL}, 9.3 \mathrm{pg} / \mathrm{mL}, 1.7 \mathrm{pg} / \mathrm{mL}, 1.8$ $\mathrm{pg} / \mathrm{mL}, 20.2 \mathrm{pg} / \mathrm{mL}, 1.2 \mathrm{pg} / \mathrm{mL}, 0.4 \mathrm{pg} / \mathrm{mL}$, and $9.9 \mathrm{pg} / \mathrm{mL}$ respectively. All the standards and samples were assayed in a duplicate test.

\section{Statistical Analysis}

Statistical analyses were performed using the Standard Package for Social Sciences version 24.0 software (IBM Corp, Armonk, NY, USA). The values were presented with mean \pm standard deviation $(\mathrm{M} \pm \mathrm{SD})$. With regards to demographic and clinical characteristics, the Chi-square tests were used for categorical data and the Student's $t$-tests were used for continuous data. Due to irregular distribution, cytokine analyses were performed using the Spearman correlations and non-parametric tests, including the Mann-Whitney $U$-tests and the Wilcoxon signed rank tests. For stepwise linear multiple regression, age, gender, $\mathrm{BMI}$, and the olanzapine equivalent dose were entered as covariates. All the tests were two-tailed and the statistical significance was declared at $\mathrm{p}<0.05$.

\section{Results}

\section{Demographic and Clinical Details of All Subjects}

The demographic and clinical characteristics of all the patients and healthy individuals are presented in Table 1.
We recruited 71 patients in this study, including 35 patients with $\mathrm{SCz}$ (20 males and 15 females with a mean age of $26.1 \pm 9.7$ years) and 36 healthy control subjects (22 males and 14 females with a mean age of $25.8 \pm 5.1$ years). No statistical significance was found in terms of the sex, smoking status, drinking status, age, and BMI between the healthy controls and the schizophrenics at the baseline $(p>0.05)$. However, after a 6-month course of antipsychotic treatment, the BMI demonstrated a significant increase as compared to the baseline value $(\mathrm{p}<0.001)$.

Regarding clinical information, the CGI scores, PANSS total scores, and the scores of five PANSS subscales were significantly reduced at the 6-month follow-up as compared to their baseline value $(p<0.001)$.

\section{Cytokine Concentrations}

As presented in Table 2, the serum levels of IL-1 $\beta$, IL-4, IL-6, and IL-8 levels were significantly increased in schizophrenic patients than in healthy controls $(\mathrm{p}=0.001$, $\mathrm{p}=0.007, \mathrm{p}<0.001, \mathrm{p}<0.001$, respectively). At the 6-month follow up, the IL-8 level had significantly decreased to its normal value, as there was no difference in the IL- 8 level between the controls and the patients after follow-up ( $\mathrm{p}>0.05)$. IL1 $\beta$, IL-4, and IL-6 levels were slightly reduced, but still elevated in the patients than in the controls after follow-up $(p=0.001, p<0.001, p=0.031$, respectively). Furthermore, the levels of TNF- $\alpha$ and CCL-2 dropped in the patients than in controls at baseline during the follow-up $(\mathrm{p}=0.012, \mathrm{p}=0.004$, respectively). This result indicated that a higher level of inflammation existed in patients with $\mathrm{SCz}$ than in healthy individuals, and the inflammation level decreased after treatment.

\section{Correlations of Cytokine Levels at Baseline and Clinical Symptom Severity}

Correlation analyses were performed to assess the serum cytokine concentrations of schizophrenic patients with PANSS scale scores at the baseline to demonstrate the relationship between the inflammation level and clinical symptom severity of patients with SCz. Table 3 shows that the IL-6 level was significantly associated with negative symptom score on the PANSS subscale $(\rho=0.470, \mathrm{p}=0.004)$. This indicated that as the concentration of IL-6 increased, the negative symptoms of $\mathrm{SCz}$ worsened. 
Table I Demographic and Clinical Details of Healthy Controls and Patients

\begin{tabular}{|c|c|c|c|c|c|}
\hline & \multirow{2}{*}{$\begin{array}{l}\mathrm{HC} \\
(n=36)\end{array}$} & \multicolumn{2}{|l|}{$\mathrm{SCz}$} & \multicolumn{2}{|l|}{$\chi^{2} / \mathrm{t}(\mathrm{p})$} \\
\hline & & $\begin{array}{l}\text { Baseline } \\
(n=35)\end{array}$ & $\begin{array}{l}\text { Follow-up } \\
(n=35)\end{array}$ & (SCz vs HC baseline) & (SCz Baseline vs follow-up) \\
\hline \multicolumn{6}{|l|}{ Gender, n } \\
\hline Male/Female & $22 / 14$ & $20 / 15$ & $20 / 15$ & $0.116(0.81 \mathrm{I})$ & \\
\hline \multicolumn{6}{|l|}{ Smoking, $\mathrm{n}$} \\
\hline Smoker & 11 & 4 & 4 & $2.833(0.079)$ & \\
\hline Nonsmoker & 25 & 31 & 31 & & \\
\hline \multicolumn{6}{|l|}{ Drinking, $\mathrm{n}$} \\
\hline Drinker & $\mathrm{I}$ & 1 & I & $0.000(1.000)$ & \\
\hline Nondrinker & 35 & 34 & 34 & & \\
\hline Age (years $\pm S D$ ) & $25.8 \pm 5.1$ & $26.1 \pm 9.7$ & $26.1 \pm 9.7$ & $0.138(0.891)$ & \\
\hline $\mathrm{BMI}( \pm \mathrm{SD})$ & $21.53 \pm 3.6$ & $21.0 \pm 3.5$ & $23.9 \pm 3.3$ & $-0.583(0.562)$ & $-6.104(<0.001)^{* *}$ \\
\hline PANSS score & & $85.8 \pm 20.0$ & $47.7 \pm 13.9$ & & II.778 $(<0.00 \mathrm{I})^{* *}$ \\
\hline Positive symptom $( \pm S D)$ & & $12.5 \pm 4.2$ & $5.1 \pm 2.4$ & & $10.035(<0.001)^{* *}$ \\
\hline Negative symptom $( \pm S D)$ & & $20.1 \pm 6.2$ & $14.4 \pm 6.9$ & & $5.740(<0.00 \mathrm{I})^{* *}$ \\
\hline Cognitive defect $( \pm S D)$ & & $9.8 \pm 3.4$ & $4.7 \pm 1.5$ & & $7.381(<0.001)^{* *}$ \\
\hline Excited symptom ( $\pm \mathrm{SD})$ & & $1 \mathrm{I} .0 \pm 4.6$ & $4.9 \pm 1.8$ & & $4.957(<0.001)^{* *}$ \\
\hline Depressive symptom( $\pm \mathrm{SD})$ & & $7.4 \pm 2.7$ & $5.1 \pm 2.0$ & & $8.780(<0.001)^{* *}$ \\
\hline $\mathrm{CGI}( \pm \mathrm{SD})$ & & $4.9 \pm 0.8$ & $2.8 \pm 1.0$ & & $12.234(<0.001)^{* *}$ \\
\hline $\begin{array}{l}\text { Antipsychotic dose } \\
\text { (olanzapine equivalents, } \mathrm{mg} / \text { day } \pm \mathrm{SD} \text { ) }\end{array}$ & & $8.19 \pm 7.73$ & & & \\
\hline
\end{tabular}

Notes: Statistical significance when $\mathrm{p} \leq 0.05$. $* * \mathrm{p} \leq 0.001$.

Abbreviations: SD, standard deviation; BMI, body mass index; PANSS, Positive and Negative Symptom Scale score; HC, healthy controls; SCz, schizophrenia.

Table 2 Comparison of Cytokine Levels Between Schizophrenic Patients and Healthy Controls

\begin{tabular}{|c|c|c|c|c|c|c|}
\hline & \multirow[t]{2}{*}{ HC } & \multicolumn{2}{|l|}{ SCz } & \multicolumn{3}{|l|}{ Z-value (p) } \\
\hline & & Baseline & Follow-up & $\begin{array}{l}\text { (SCz Baseline vs } \\
\text { HC) }\end{array}$ & $\begin{array}{l}\text { (SCz Baseline vs } \\
\text { Follow-up) }\end{array}$ & $\begin{array}{l}\text { (SCz Follow-up vs } \\
\mathrm{HC} \text { ) }\end{array}$ \\
\hline TNF- $\alpha, p g / m L$ & $3.0 \pm 1.5$ & $3.2 \pm 1.5$ & $2.7 \pm 1.2$ & $-0.558(0.577)$ & $-2.502(0.012)^{*}$ & $-1.013(0.311)$ \\
\hline IL-6, pg/mL & $1.0 \pm 0.8$ & $2.1 \pm 2.3$ & $2.1 \pm 3.6$ & $-3.372(0.001)^{*}$ & $-1.196(0.232)$ & $-2.162(0.031)^{*}$ \\
\hline IL-8, pg/mL & $10.5 \pm 9.9$ & $40.6 \pm 55.8$ & $21.6 \pm 35.8$ & $-2.680(0.007)^{*}$ & $-1.513(0.130)$ & $-0.846(0.398)$ \\
\hline $\mathrm{CCL} 2, \mathrm{pg} / \mathrm{mL}$ & $218.6 \pm 78.2$ & $234.2 \pm 82.0$ & $205.0 \pm 78.9$ & $-0.920(0.357)$ & $-2.886(0.004)^{*}$ & $-0.650(0.516)$ \\
\hline IL-I $\beta, p g / m L$ & $1.7 \pm 0.7$ & $2.4 \pm 0.7$ & $2.3 \pm 0.8$ & $-3.602(<0.00 \mathrm{I})^{* *}$ & $-0.674(0.500)$ & $-3.416(0.001)^{*}$ \\
\hline IFN- $\gamma, p g / m L$ & $13.4 \pm 11.3$ & $12.3 \pm 9.3$ & $12.4 \pm 8.2$ & $-0.207(0.836)$ & $-0.336(0.737)$ & $-0.155(0.877)$ \\
\hline IL-4, pg/mL & $12.5 \pm 17.0$ & $38.6 \pm 15.5$ & $34.9 \pm 15.3$ & $-5.516(<0.001)^{* *}$ & $-1.67 \mid(0.095)$ & $-5.222(<0.001)^{* *}$ \\
\hline IL-12 p70, pg/mL & $24.0 \pm 18.2$ & $17.0 \pm 5.0$ & $16.9 \pm 8.5$ & $-1.715(0.086)$ & $-1.529(0.126)$ & $-1.847(0.065)$ \\
\hline
\end{tabular}

Notes: Statistical significance when $\mathrm{p} \leq 0.05$. $* \mathrm{p} \leq 0.05$. ** $\mathrm{p} \leq 0.00 \mathrm{I}$.

Abbreviations: TNF, tumor necrosis factor; IL, interleukin; CCL, C-C motif chemokine ligand; IFN, interferon.

Predictive Factors of Clinical Therapeutic Effects

The regression model in Table 4 shows that serum cytokine levels at the baseline can be used as predictors of clinical therapeutic effects at the 6-month follow-up. The reductive ratio of the PANSS scale scores were used to reflect the effects of the clinical antipsychotic treatment. According to the regression model, at the 6-month follow-up, the higher IL6 level $(p=0.027)$ and lower IL-8 level $(p=0.035)$ pre dicted a better therapeutic effect on negative symptoms (Figure 1A,B). In addition, a higher IL-6 level $(\mathrm{p}=0.002)$ predicted a reduced improvement in depressive symptoms 
Table 3 Correlation Analysis Between Cytokine Concentrations of Patients with Schizophrenia at Baseline and Clinical Symptom Severity

\begin{tabular}{|c|c|c|c|c|c|c|}
\hline & \multicolumn{6}{|c|}{ Spearman Rho (p) } \\
\hline & Total Score & $\begin{array}{l}\text { Positive } \\
\text { Symptom }\end{array}$ & $\begin{array}{l}\text { Negative } \\
\text { Symptom }\end{array}$ & $\begin{array}{l}\text { Cognitive } \\
\text { Symptom }\end{array}$ & $\begin{array}{l}\text { Excited } \\
\text { Msymptom }\end{array}$ & $\begin{array}{l}\text { Depression } \\
\text { Symptom }\end{array}$ \\
\hline TNF- $\alpha, p g / m L$ & 0.001 (0.994) & $-0.024(0.891)$ & $0.321(0.060)$ & $-0.233(0.177)$ & $-0.153(0.379)$ & $-0.219(0.207)$ \\
\hline IL-6, pg/mL & $0.054(0.756)$ & $-0.148(0.396)$ & $0.470(0.004)^{*}$ & $0.013(0.942)$ & -0.181 (0.299) & $-0.150(0.390)$ \\
\hline IL-8, pg/mL & $-0.191(0.271)$ & $-0.040(0.817)$ & $-0.011(0.952)$ & $-0.154(0.378)$ & $-0.178(0.307)$ & $-0.161(0.356)$ \\
\hline $\mathrm{CCL} 2, \mathrm{pg} / \mathrm{mL}$ & $0.050(0.775)$ & $-0.108(0.535)$ & $0.197(0.256)$ & $0.079(0.652)$ & $-0.131(0.453)$ & $0.032(0.856)$ \\
\hline IL-I $\beta, p g / m L$ & $0.048(0.786)$ & $0.032(0.853)$ & $0.230(0.183)$ & $-0.200(0.249)$ & $-0.108(0.537)$ & $-0.093(0.593)$ \\
\hline $\mathrm{IFN}-\gamma, \mathrm{pg} / \mathrm{mL}$ & $0.171(0.325)$ & $0.030(0.864)$ & $0.326(0.056)$ & $-0.060(0.732)$ & $0.002(0.990)$ & $-0.004(0.983)$ \\
\hline IL-4, pg/mL & $-0.059(0.737)$ & $-0.089(0.61 \mathrm{I})$ & $0.162(0.354)$ & $-0.092(0.599)$ & $-0.162(0.353)$ & $-0.006(0.972)$ \\
\hline IL-12 p70, pg/mL & $-0.036(0.839)$ & $-0.064(0.713)$ & $-0.033(0.85 I)$ & $0.066(0.706)$ & $-0.059(0.738)$ & $0.246(0.154)$ \\
\hline
\end{tabular}

Notes: Statistical significance when $\mathrm{p} \leq 0.05 . * \mathrm{p} \leq 0.05$.

(Figure 1D). Finally, a higher interferon (IFN)- $\gamma$ level predicted a lower therapeutic effect for excitatory symptoms (Figure 1C).

\section{Discussion}

Our results show that immune/inflammatory reactions in $\mathrm{SCz}$ patients are stronger as compared to the inflammatory pathways of a healthy body. Cytokine levels have been found to be closely related to the status and severity of the disease. In our study, four of eight cytokines (IL-1 $\beta$, IL-4, IL-6, and IL-8) were significantly elevated in the patients than in controls. This result was consistent with those of previous studies. ${ }^{23-25}$ It is intriguing that IL-1 $\beta$, IL-6, and IL-8, which were found to be elevated in the acute stage of $\mathrm{SCz}$, are modulated through $\mathrm{NF}-\mathrm{kB}$, a signaling pathway that is commonly activated in inflammatory diseases. ${ }^{26-28}$ This indicates that people with SCz may undergo a factor of immune system activation at the acute stage of the disease. ${ }^{23}$ At the 6-month follow-up, seven cytokines, except
IL-6, had reduced as compared to their baseline values, of which IL-8 had reduced most significantly, that it, it attained normal levels after clinical remission. This finding suggests that IL-8 is likely to be a state-associated marker. However, Miller et al found that IL-6 levels significantly decreased following the treatment of acute $\mathrm{SCz}^{23}$ which is inconsistent with our research. This may be due to the influences of confounding factors, such as age, BMI, drinking habits, and smoking status. Furthermore, our results showed that the severity of negative symptoms was strongly related to IL-6 levels; this finding is accordant with that of a previous study. ${ }^{29}$

We also found a statistically significant correlation between the clinical therapeutic effect as measured by PANSS score reductive ratio and the peripheral blood levels of cytokines by establishing regression models of the two entities. Negative and depressive symptoms have been suggested to be associated with real-world functioning and disability in some studies. ${ }^{30,31}$ Negative symptoms seem to have

Table 4 Linear Regression Models for Predictors of Clinical Therapeutic Effects

\begin{tabular}{|c|c|c|c|c|c|c|}
\hline & \multicolumn{6}{|c|}{ Model } \\
\hline & $\mathbf{R}^{2}$ & dF & F-value (p) & Beta & t-value $(p)$ & VIF \\
\hline PANSS negative symptom subscale score reductive ratio & 0.299 & 6 & $3.415(0.012)^{*}$ & & & \\
\hline IL-6 & & & & 0.418 & $2.338(0.027)^{*}$ & $1.55 \mathrm{I}$ \\
\hline IL-8 & & & & -0.373 & $-2.214(0.035)^{*}$ & 1.375 \\
\hline PANSS excited symptom subscale score reductive ratio & 0.248 & 5 & $3.244(0.019)^{*}$ & & & \\
\hline IFN- $\gamma$ & & & & -0.679 & $-3.927(<0.001)^{* *}$ & 1.354 \\
\hline PANSS depressive symptom subscale score reductive ratio & 0.279 & 5 & $3.630(0.011)^{*}$ & & & \\
\hline IL-6 & & & & -0.534 & $-3.394(0.002)^{*}$ & 1.166 \\
\hline
\end{tabular}

Notes: Statistical significance when $p \leq 0.05$. ${ }^{*} p \leq 0.05$. ${ }^{* *} p \leq 0.001$.

Abbreviation: VIF, multicollinearity. 

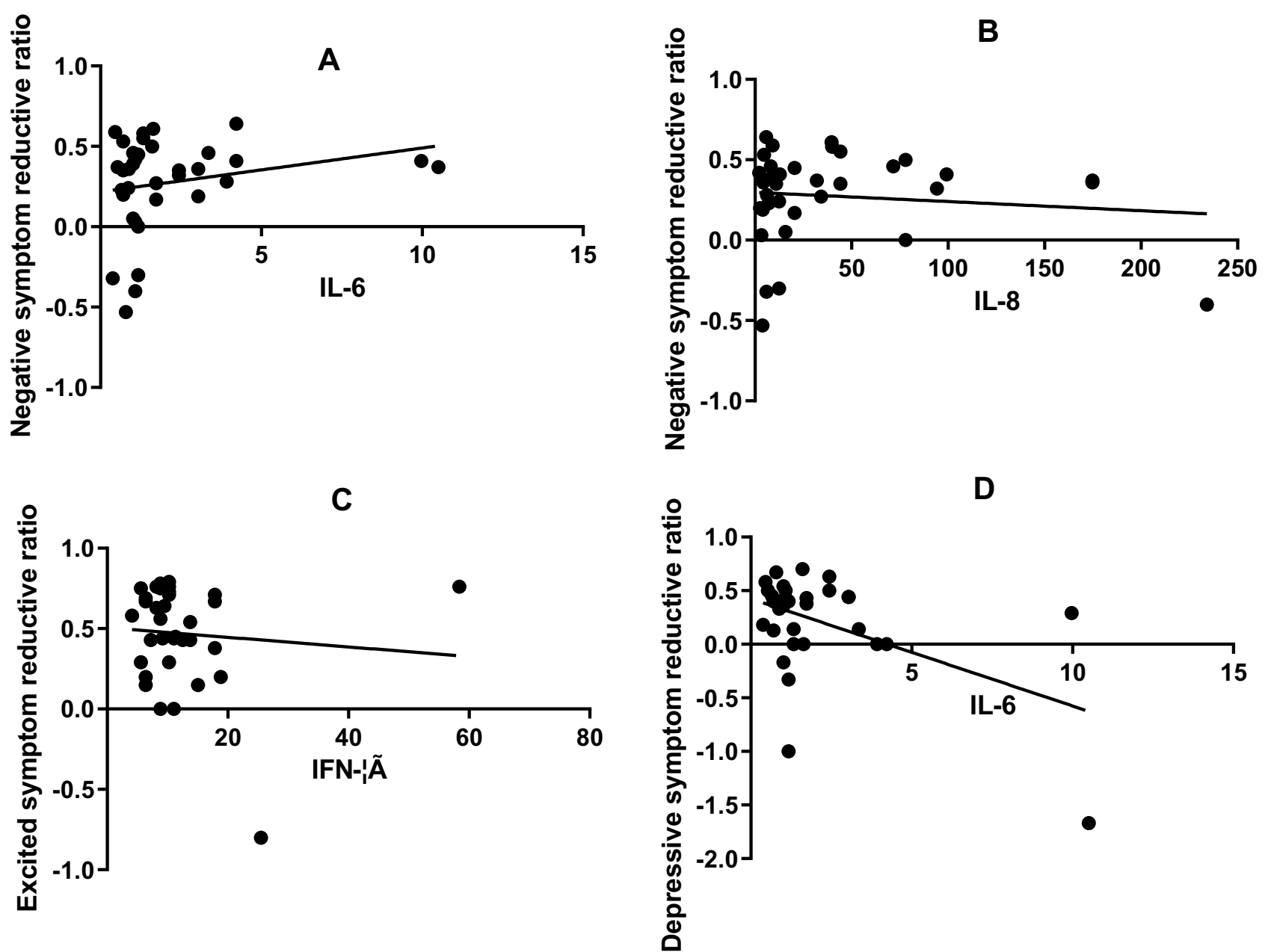

Figure I Correlations between cytokine levels at baseline and PANSS scores reductive ratio. (A) Correlations between IL-6 levels at baseline and PANSS negative symptom scores reductive ratio. (B) Correlations between IL-8 levels at baseline and PANSS negative symptom scores reductive ratio. (C) Correlations between IFN- $\gamma$ levels at baseline and PANSS excitatory symptom scores reductive ratio. (D) Correlations between IL-6 levels at baseline and PANSS depressive symptom scores reductive ratio.

a greater impact on interpersonal and social functioning outcomes $^{32}$ and these symptoms usually do not respond to conventional antipsychotic medication. ${ }^{33}$ Depressive symptoms can occur in all stages of $\mathrm{SCz}$ and are related to bad social and cognitive performance and poor response to medications. ${ }^{34}$ Additionally, depression is an important predictor of disability and suicidal risk in SCz. ${ }^{13}$ Positive symptoms can affect specific areas, such as self-care and aggressive behavior, but tend to be clinically relived through antipsychotic treatment. ${ }^{35}$ Thus, it is interesting to see whether blood cytokine levels in early stage of $\mathrm{SCz}$ can predict the final therapeutic effect of these symptoms and whether they can reduce negative and depressive symptoms that may lead to a reduction in the level of disability.

Our research showed that higher IL-6 levels predict a reduced improvement in depressive symptoms. The cytokine IL-6 is released from activated monocytes and Th2 lymphocytes. $^{36}$ It acts as a mediator of the peripheral proinflammatory response and might be the most important cytokine in microglia activation and inflammation. Peripheral IL-6 levels have been confirmed to be associated with a decrease in hippocampal gray matter volume ${ }^{37}$ due to their inhibitive effect on hippocampal neurogenesis, while impaired neurogenesis is known to recover under the onfluence of IL- $6 .{ }^{38}$ Many studies have found that plasma levels of IL-6 were elevated in major depression patients as compared to healthy subjects. ${ }^{39,40}$ In combination with the finding mentioned above, we postulate that increased levels of IL-6 during the acute stage of SCz may be considered a predictor of a poor treatment response to depressive symptoms, and it is necessary to study whether intervention in the early stage can reduce the manifestation of such symptoms.

Another important result is that higher IL-8 levels were found to be associated with poorer improvement in negative symptoms. One study demonstrated that negative symptoms are the main determining factors of poor real-world functioning 
in patients with $\mathrm{SCz}^{41}$ and the remission of negative symptoms contribute to functional recovery. ${ }^{42} \mathrm{IL}-8$ is an $8 \mathrm{kDa}$ protein and a member of a family of soluble molecules called chemokines, which plays a central role in the immune response. IL-8 can regulate neutrophil traffic during acute inflammatory responses, lead them to tissues, and discharge lysosomal enzymes, which results in the release of oxygen free radicals. ${ }^{43}$ One study showed that fetal exposure to high levels of maternal IL-8 could lead to structural brain alterations and increased risk for $\mathrm{SCz}^{44}$ In agreement with these findings, our results suggest that IL-8 may participate in the pathophysiological changes leading to $\mathrm{SCz}$ and the IL- 8 levels at baseline could be considered as biomarkers for evaluating the therapeutic effect on negative symptoms of SCz.

These findings provide evidence to predict the efficacy of residual symptoms for $\mathrm{SCz}$ and offer potential possibilities for the remission of these symptoms. Some scholars have suggested that inflammatory cytokines can be used as emerging therapeutic targets for $\mathrm{SCz} .{ }^{45,46}$ One study demonstrated that when schizophrenic patients were treated with celecoxib combined with antipsychotic drugs, the celecoxib group showed significantly greater therapeutic effects. ${ }^{19}$ Furthermore, a recent study evaluated tocilizumab, a humanized IL-6 receptor monoclonal antibody, as an adjunctive treatment. It was found that treating schizophrenic patients with tocilizumab could significantly improve their cognitive function. ${ }^{47}$ Meanwhile, Girgis et al conducted an randomized controlled trial study and found no evidence that IL-6 receptor antibodies affect the behavioral prognosis of SCz. ${ }^{48}$ They suggested a possible explanation: that tocilizumab lacks the ability to penetrate the central nervous system.

Further studies are needed to apply these drugs to studies with larger sample sizes and explore new methods of effective treatment. It is noteworthy that the depression factor used in our study was derived from the five-factor model of the PANSS scale. ${ }^{22}$ We did not use a specific depression scale, like the Calgary Depression scale for Schizophrenia (CDSS), for our patients. Therefore, the association between IL-6 and depressive symptoms in SCz deserves further investigation using CDSS in a prospective study.

There are several strengths to the present study. First, our study is longitudinal and prospective, which reduces the influence of bias. Second, possible confounders (BMI, smoking status, and drinking status) that may have interfered with the immune system and inflammatory response were well-controlled, which increased the reliability of our outcomes. However, our research also had some limitations, such as the relatively modest sample size. Additionally, while many studies have reported that antipsychotic medications may affect the plasma cytokine levels, ${ }^{49,50}$ it is still unclear whether the changes in cytokine levels were an outcome of symptom improvement or resulted from the response of the antipsychotic medications.

\section{Conclusions}

The serum levels of inflammatory markers were higher in patients with $\mathrm{SCz}$ than in healthy controls. These markers can be considered accurate predictors of therapeutic effects in patients with $\mathrm{SCz}$.

\section{Acknowledgment}

The authors thank all the participants in this study for their support.

\section{Funding}

This study was supported by the National Natural Science Foundation of China (no. 81771471), Shaanxi Province Innovation Capacity Support Program (no. 2017KCT-20), and Shaanxi Provincial Key Research and Development Program (no. 2017ZDXM-SF-25-6).

\section{Disclosure}

All authors report no conflicts of interest in this work.

\section{References}

1. Andrade C. Anti-inflammatory strategies in the treatment of schizophrenia. Expert Rev Clin Pharmacol. 2016;9(2):161-163. doi:10.1586/17512433.2016.1095086

2. Smyth AM, Lawrie SM. The neuroimmunology of schizophrenia. Clin Psychopharmacol Neurosci. 2013;11(3):107-117. doi:10.9758/cpn.20 13.11.3.107

3. Kahn RS, Sommer IE, Murray RM, et al. Schizophrenia. Nat Rev Dis Primers. 2015;1. doi:10.1038/nrdp.2015.67.

4. McGrath J, Saha S, Chant D, et al. Schizophrenia: a concise overview of incidence, prevalence, and mortality. Epidemiol Rev. 2008;30 (1):67-76. doi:10.1093/epirev/mxn001

5. Harvey PD, Strassnig MT. Cognition and disability in schizophrenia: cognition-related skills deficits and decision-making challenges add to morbidity. World Psychiatry. 2019;18(2):165-167. doi:10.1002/wps.v18.2

6. Charlson FJ, Ferrari AJ, Santomauro DF, et al. Global epidemiology and burden of schizophrenia: findings from the global burden of disease study 2016. Schizophr Bull. 2018;44(6):1195-1203. doi:10. 1093/schbul/sby058

7. Harvey PD, Strassnig M. Predicting the severity of everyday functional disability in people with schizophrenia: cognitive deficits, functional capacity, symptoms, and health status. World Psychiatry. 2012;11(2):73-79. doi:10.1016/j.wpsyc.2012.05.004

8. Keshavan MS, Lawler AN, Nasrallah HA, et al. New drug developments in psychosis: challenges, opportunities and strategies. Prog Neurobiol. 2017;152:3-20. doi:10.1016/j.pneurobio.2016.07.004

9. Li CY, Tao H, Yang X, et al. Assessment of a combination of serum proteins as potential biomarkers to clinically predict schizophrenia. Int J Med Sci. 2018;15(9):900-906. doi:10.7150/ijms.24346 
10. Orsolini L, Sarchione F, Vellante F, et al. Protein-C reactive as biomarker predictor of schizophrenia phases of illness? A systematic review. Curr Neuropharmacol. 2018;16(5):583-606. doi:10.2174/1570159X16666180119144538

11. Mednick SA, Machon RA, Huttunen MO, Bonett D. Adult schizophrenia following prenatal exposure to an influenza epidemic. Arch Gen Psychiatry. 1988;45(2):189-192. doi:10.1001/archpsyc.1988.018 00260109013

12. Cullen AE, Holmes S, Pollak TA, et al. Associations between non-neurological autoimmune disorders and psychosis: a meta-analysis. Biol Psychiatry. 2019;85(1):35-48. doi:10.1016/j. biopsych.2018.06.016

13. Bagaric D, Brečić P, Ostojić D, et al. The relationship between depressive syndrome and suicidal risk in patients with acute schizophrenia. Croat Med J. 2013;54(5):436-443. doi:10.3325/ cmj.2013.54.436

14. Ripke S, Neale BM, Corvin A. Biological insights from 108 shizophrenia-associated genetic loci. Nature. 2014;511(7510):421-+.

15. Benros ME, Mortensen PB, Eaton WW. Autoimmune diseases and infections as risk factors for schizophrenia. Neuroimmunomodulation Health Dis. 2012;1262:56-66.

16. Lesh TA, Careaga M, Rose DR, et al. Cytokine alterations in first-episode schizophrenia and bipolar disorder: relationships to brain structure and symptoms. J Neuroinflammation. 2018;15. doi:10.1186/s12974-018-1197-2.

17. Fillman SG, Weickert TW, Lenroot RK, et al. Elevated peripheral cytokines characterize a subgroup of people with schizophrenia displaying poor verbal fluency and reduced Broca's area volume. Mol Psychiatry. 2016;21(8):1090-1098. doi:10.1038/mp.2015.90

18. Hoseth EZ, Ueland T, Dieset I, et al. A study of TNF pathway activation in schizophrenia and bipolar disorder in plasma and brain tissue. Schizophr Bull. 2017;43(4):881-890. doi:10.1093/schbul/ sbw183

19. Muller N, Riedel M, Scheppach C, et al. Beneficial antipsychotic effects of celecoxib add-on therapy compared to risperidone alone in schizophrenia. Am J Psychiatry. 2002;159(6):1029-1034. doi:10.11 76/appi.ajp.159.6.1029

20. Sommer IE, de Witte L, Begemann $M$, et al. Nonsteroidal anti-inflammatory drugs in schizophrenia: ready for practice or a good start? A meta-analysis. J Clin Psychiatry. 2012;73(4):414-419. doi:10.4088/JCP.10r06823

21. Tang HP, Panemangalore R, Yarde M, et al. 384-well multiplexed luminex cytokine assays for lead optimization. J Biomol Screen. 2016;21(6):548-555. doi:10.1177/1087057116644164

22. Wallwork RS, Fortgang R, Hashimoto R, et al. Searching for a consensus five-factor model of the positive and negative syndrome scale for schizophrenia. Schizophr Res. 2012;137(1-3):246-250. doi:10.1016/j.schres.2012.01.031

23. Goldsmith DR, Rapaport MH, Miller BJ. A meta-analysis of blood cytokine network alterations in psychiatric patients: comparisons between schizophrenia, bipolar disorder and depression. Mol Psychiatry. 2016;21(12):1696-1709. doi:10.1038/mp.2016.3

24. Lee EE, Hong S, Martin AS, et al. Inflammation in schizophrenia: cytokine levels and their relationships to demographic and clinical variables. Am J Geriatr Psychiatry. 2017;25(1):50-61. doi:10.1016/j. jagp.2016.09.009

25. Dahan S, Bragazzi NL, Yogev A, et al. The relationship between serum cytokine levels and degree of psychosis in patients with schizophrenia. Psychiatry Res. 2018;268:467-472. doi:10.1016/j. psychres.2018.07.041

26. Akira S, Kishimoto T, Akira S. NF-IL6 and NF-kappa B in cytokine gene regulation. Adv Immunol. 1997;65:1-46.

27. Thiel G, Ulrich M, Mukaida N, et al. Resveratrol stimulation induces interleukin-8 gene transcription via NF-kappa B. Pharmacol Res. 2018;134:238-245. doi:10.1016/j.phrs.2018.07.003
28. Thomas R. The TRAF6-NF kappa B signaling pathway in autoimmunity: not just inflammation. Arthritis Res Ther. 2005;7(4):1 70-173. doi:10.1186/ar1784

29. Kim YK, Kim L, Lee MS. Relationships between interleukins, neurotransmitters and psychopathology in drug-free male schizophrenics. Schizophr Res. 2000;44(3):165-175. doi:10.1016/S0920-9964(99)00171-1

30. Strassnig M, Bowie C, Pinkham AE, et al. Which levels of cognitive impairments and negative symptoms are related to functional deficits in schizophrenia? J Psychiatr Res. 2018;104:124-129. doi:10.1016/j. jpsychires.2018.06.018

31. Harvey PD. Assessing disability in schizophrenia: tools and contributors. J Clin Psychiatr. 2014;75(10):e27. doi:10.4088/JCP.13049tx5c

32. Rocca $\mathrm{P}$, Montemagni $\mathrm{C}$, Zappia $\mathrm{S}$, Piterà R, Sigaudo $\mathrm{M}$, Bogetto $\mathrm{F}$. Negative symptoms and everyday functioning in schizophrenia: a cross-sectional study in a real world-setting. Psychiatry Res. 2014;218(3):284-289. doi:10.1016/j.psychres.2014.04.018

33. Davis MC, Horan WP, Marder SR. Psychopharmacology of the negative symptoms: current status and prospects for progress. Eur Neuropsychopharmacol. 2014;24(5):788-799. doi:10.1016/j.euroneuro. 2013.10.010

34. Siris SG. Depression in schizophrenia: perspective in the era of "Atypical" antipsychotic agents. Am J Psychiatry. 2000;157 (9):1379-1389. doi:10.1176/appi.ajp.157.9.1379

35. Menendez-Miranda I, Garcia-Portilla MP, Garcia-Alvarez L, et al. Predictive factors of functional capacity and real-world functioning in patients with schizophrenia. Eur Psychiatry. 2015;30(5):622-627. doi:10.1016/j.eurpsy.2014.12.011

36. Maes M, Stevens W, Scharpe S. Seasonal variation in peripheral blood leukocyte subsets and in serum interleukin-6, and soluble interleukin-2 and -6 receptor concentrations in normal volunteers. Experientia. 1994;50(9):821-829.

37. Marsland AL, Gianaros PJ, Abramowitch SM, Manuck SB, Hariri AR. Interleukin-6 covaries inversely with hippocampal grey matter volume in middle-aged adults. Biol Psychiatry. 2008;64 (6):484-490. doi:10.1016/j.biopsych.2008.04.016

38. Monje ML, Toda H, Palmer TD. Inflammatory blockade restores adult hippocampal neurogenesis. Science. 2003;302(5651):1760-17 65. doi: $10.1126 /$ science. 1088417

39. Dowlati Y, Herrmann N, Swardfager W, et al. A meta-analysis of cytokines in major depression. Biol Psychiatry. 2010;67(5):446-457. doi:10.1016/j.biopsych.2009.09.033

40. Young JJ, Bruno D, Pomara N. A review of the relationship between proinflammatory cytokines and major depressive disorder. $J$ Affect Disord. 2014;169:15-20. doi:10.1016/j.jad.2014.07.032

41. Gonzalez-Blanco L, Garcia-Portilla MP, Dal Santo F, et al. Predicting real-world functioning in outpatients with schizophrenia: role of inflammation and psychopathology. Psychiatry Res. 2019;280:11 2509. doi:10.1016/j.psychres.2019.112509

42. Santesteban-Echarri O, Paino M, Rice S, et al. Predictors of functional recovery in first-episode psychosis: a systematic review and meta-analysis of longitudinal studies. Clin Psychol Rev. 2017;58:59-75. doi:10.1016/j.cpr.2017.09.007

43. Brown AS, Hooton J, Schaefer CA, et al. Elevated maternal interleukin-8 levels and risk of schizophrenia in adult offspring. $\mathrm{Am}$ J Psychiatry. 2004;161(5):889-895. doi:10.1176/appi.ajp.161.5.889

44. Ellman LM, Deicken RF, Vinogradov S, et al. Structural brain alterations in schizophrenia following fetal exposure to the inflammatory cytokine interleukin-8. Schizophr Res. 2010;121(1-3):46-54. doi:10. 1016/j.schres.2010.05.014

45. Girgis RR, Kumar SS, Brown AS. The cytokine model of schizophrenia: emerging therapeutic strategies. Biol Psychiatry. 2014;75 (4):292-299. doi:10.1016/j.biopsych.2013.12.002

46. Kogan S, Ospina LH, Kimhy D. Inflammation in individuals with schizophrenia - Implications for neurocognition and daily function. Brain Behav Immun. 2018;74:296-299. doi:10.1016/j.bbi.2018.09.016 
47. Miller BJ, Dias JK, Lemos HP, et al. An open-label, pilot trial of adjunctive tocilizumab in schizophrenia. J Clin Psychiatry. 2016;77 (2):275-276. doi:10.4088/JCP.15109920

48. Girgis RR, Ciarleglio A, Choo T, et al. A randomized, double-blind, placebo-controlled clinical trial of tocilizumab, an interleukin-6 receptor antibody, for residual symptoms in schizophrenia. Neuropsychopharmacology. 2018;43(6):1317-1323. doi:10.1038/ npp. 2017.258
49. Maes M, Chiavetto LB, Bignotti S, et al. Effects of atypical antipsychotics on the inflammatory response system in schizophrenic patients resistant to treatment with typical neuroleptics. Eur Neuropsychopharmacol. 2000;10(2):119-124. doi:10.1016/S0924-97 7X(99)00062-0

50. Tourjman V, Kouassi É, Koué M-Ė, et al. Antipsychotics' effects on blood levels of cytokines in schizophrenia: a meta-analysis. Schizophr Res. 2013;151(1-3):43-47. doi:10.1016/j.schres.2013.10.011

\section{Publish your work in this journal}

Neuropsychiatric Disease and Treatment is an international, peerreviewed journal of clinical therapeutics and pharmacology focusing on concise rapid reporting of clinical or pre-clinical studies on a range of neuropsychiatric and neurological disorders. This journal is indexed on PubMed Central, the 'PsycINFO' database and CAS, and is the official journal of The International Neuropsychiatric Association (INA). The manuscript management system is completely online and includes a very quick and fair peer-review system, which is all easy to use. Visit http://www.dovepress.com/testimonials.php to read real quotes from published authors. 